\title{
REGIONAL EXPONENTIAL GENERAL OBSERVABILITY IN DISTRIBUTED PARAMETER SYSTEMS
}

\author{
Raheam A. Al-Saphory ${ }^{1 *}$, andZainab A. Jaafar ${ }^{1}$
}

${ }^{1}$ Department of Mathematics, College of Education for Pure Sciences, Tikrit University, Tikrit, IRAQ.

\section{ABSTRACT}

The purpose of this paper is to explore the concept of regional exponential general observability for a class of distributed parameter systems, in connection with the regional strategic sensors. Then, we give characterization of the strategic sensors in order that exponential regional general observability can be achieved. Furthermore, we apply these results to one and two infinite dimensional systems, and various cases of sensors are considered and discussed. We also show that there exists a dynamical system for diffusion system is not exponentially general observable in the usual sense, but it may be regionally exponentially general observable.

Keywords: Strategic sensors, $\omega_{E_{G}}$-estimator, $\omega_{E_{G}}$ - observability, $\omega_{E_{G}}$ - strategic sensor, diffusion systems.

\section{Academic Discipline and Sub-Disciplines:}

Applied Mathematics/ Control Systems and Analysis

\section{AMS SUBJECT CLASSIFICATION:}

93АЗ0; 93В07; 93В28; 93С05; 93С28.

\section{Council for Innovative Research}

Peer Review Research Publishing System

Journal: JOURNAL OF ADVANCES IN MATHEMATICS

Vol . 11, No. 4

www.cirjam.com, editoriam@gmail.com 


\section{INTODUCTION}

The theory of observer was introduced by Luenberger (1966) [26] and has been generalized to system described by semigroup operators governed by partial differential infinite dimensional equations in Hilbert state space (Gressang and Lamont 1975) [25]. In system theory, the exponential observability is related to the possibility to estimate the state from knowledge of the state estimator system and output function [15]. The concept of actuators and sensors are introduced and applied to the controllability, observability and exponential estimator of systems described by partial differential equations as in [16-17].

The notion of regional analysis was extended by El Jai et al. [4, 6, 12, 21-23].The study of this notion motivated by certain concrete-real problem, in thermic, mechanic, environment or in isentropic superdense star as in [9, 22, 23-24]. The concept of regional asymptotic and exponential analysis was introduced recently by Al-Saphory and El Jai et al. in [1-11], consists in studying the behaviour of the system not in the entire domain $\Omega$ but only on particular region $\omega$ inside the domain or on it is boundary. The aim of this paper is to extend some results related to regional exponential observability and strategic sensor to the general case as in ref.s [3, 7]. We consider a class of distributed system and we explore various results connected with the different types of measurement, domains, and boundary conditions.

The purpose behind to develop this paper is that when we have fundamental aspects of a system knowledge, which developed by theoreticians from a mathematical point of view, and there is very often a wide gap between this and a concrete comprehension of the system [15-16]. Thus, sensors and actuators can play a fundamental role in the understanding of any real system [17-20].It is now time to link some of this theoretical works with concrete considerations of input-output problems. The main reason for discovering this concept is that it provides a means to deal with some physical problems concern the model of single room shown in (Figure 1) below.

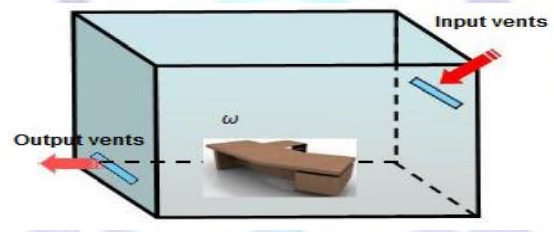

Fig. 1: Room observation problem $\Omega$, workspace $\omega$, and input-output vents

Here the goal to design the room (locate vents, place sensors, etc. ...)in order to observe exponentially the room temperature near workspace (for more details see [13]). The outline of this paper is organized as follows:

Section 2 is related to define the problem statement, regional strategic sensors, definitions and characterizations. Section 3 defines the regional exponential estimator (general case) for a distributed parameter system in terms of regional detectability and strategic sensors. We discuss under which condition such a regional observability exists and give a counter-example of an $\omega$ - estimator which is not an estimator in the whole domain $\Omega$. Section 4 is related to the characterization notion of $\omega_{E_{G}}$ - observable by the use of strategic sensors. Section 5 , we illustrate applications with many situations of sensors locations in one or two dimensional systems.

\section{REGIONAL EXPONENTIAL OBSERVABILITY}

\subsection{Problem Statement}

Let $\Omega$ be an open regular bounded set of $R^{n}$, with smooth boundary $\partial \Omega$ and let $[0, T], T>0$ be a time measurement interval. Suppose that $\omega$ be a nonempty given sub-region of $\Omega$. We denote $\Theta=\Omega \times(0, \infty)$ and $\Pi=\partial \Omega \times(0, \infty)$. The considered distributed parameter system is described by the following parabolic equations:

$\left\{\begin{array}{c}\frac{\partial x}{\partial t}(\xi, t)=A x(\xi, t)+B u(t) \Theta \\ x(\xi, 0)=x_{0}(\xi) \Omega \\ x(\eta, t)=0\end{array}\right.$

augmented with the output function

$z(\xi, t)=C x(\xi, t)$ 
where $A$ is a second-order linear differential operator, which generates a strongly continuous semi-group $\left(S_{A}(t)\right)_{t \geq 0}$ on the Hilbert space $X=L^{2}(\Omega)$ and is self-adjoint with compact resolvent. The spaces $X, U$, and $\mathcal{O}$ are separable Hilbert space where $X$ is the state space, $U=L^{2}\left(0, \infty, R^{p}\right)$ is the control space, and $\mathcal{O}=L^{2}\left(0, \infty, R^{q}\right)$ is the observation space, where pand $q$ are the numbers actuators and sensors [11], see (Figure 2) which is mathematical model more general spatial case in (Figure 1).

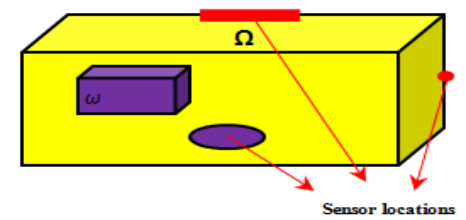

Fig. 2: The domain of $\Omega$, the subregion $\omega$, and the sensors location

The operators $B \in \mathcal{L}(U, X)$ and $C \in \mathcal{L}(X, \mathcal{O})$ depend on the structures of actuators and sensors [9,11]. Under the given assumption [19], the system (1) has a unique solution:

$x(\xi, t)=S_{A}(t) x_{0}(\xi)+\int_{0}^{t} S_{A}(t-\tau) B u(\tau) d \tau$

(3) The

problem is that how to construct an approach which observe exponential general current state in sub-region $\omega$.

\subsection{Regional Strategic Sensors}

This subsection consists of the concept of the sensors, which was coined by A. El Jai as in [16-17]. Thus, sensors can play a fundamental role in understanding of any real system. Moreover, sensors form an important link between a system and its environment [19-20]. The purpose of this subsection is to give the characterization for sensors in order that the system (1) is regionally exponentially general observable in $\omega$.

- Sensors are any couple $\left(D_{i}, g_{i}\right)_{1 \leq i \leq q}$ where $D_{i}$ denote closed subsets of $\bar{\Omega}$, which is spatial supports of sensors and $g_{i} \in L^{2}\left(D_{i}\right)$ define the spatial distributions of measurement on $\left(D_{i}\right)$.

- According to the choice of the parameters $D_{i}$ and $g_{i}$, we have various types of sensors where in the case of $q$ sensors. We shall consider $\left(D_{i}, g_{i}\right)_{1 \leq i \leq q}$ where $D_{i} \subset \Omega$, (or $\left.D_{i} \subset \partial \Omega\right)$ and $g_{i} \in L^{2}\left(D_{i}\right)$ with $D_{i} \cap D_{j}=\emptyset$ if $1 \leq i \neq j \leq q$. Then, the output function (2) may be written by the following form

where

$$
z(., t)=\left[z_{1}(., t), z_{2}(., t), \ldots, z_{q}(., t)\right]^{t_{r}}
$$

$z(\xi, t)=C x(\xi, t)=\int_{D_{i}} x(\xi, t) g_{i}(\xi) d \xi$

- Sensors may also be pointwise when $D_{i}=\left\{b_{i}\right\}$ and $g_{i}=\delta_{b_{i}}\left(x-b_{i}\right)$ where $\delta_{b_{i}}$ is Dirac mass concentrated in $b_{i}$. Then, the output function (2) can be given by the form

$z(\xi, t)=C x(\xi, t)=\int_{\Omega} x(\xi, t) \delta_{b_{i}}\left(\xi-b_{i}\right) d \xi$

- In the case of boundary zone sensor, we consider $\Omega=\Gamma$ with $\Gamma \subset \partial \Omega$ and $g \in L^{2}(\Gamma)$. Then, the output function (2) can be written in the form

$z(\xi, t)=C x(\xi, t)=\int_{\Gamma} x(\xi, t) g_{i}(\xi) d \xi$

(6) $\bullet$ In the case, of

internal pointwise sensors the operator $C$ is unbounded and some precaution must be taken in [14].

- For the following equation

$x(\xi, t)=S_{A}(t) x_{0}(\xi)(7)$ define the operators $K: X \rightarrow \mathcal{O}$

$$
x \rightarrow K(x)=C S_{A}(t) x
$$

$K(t) x_{0}(\xi)$. We denote by $K^{*}: \mathcal{O} \rightarrow X$ the adjoint of $K$ by

(8)then $z(\xi, t)=$

$K^{*} z(\xi, t)=\int_{0}^{t} S^{*}{ }_{A}(s) C^{*} z(\xi, s) d s$ 
-Consider a subdomain $\omega$ of $\Omega$ and let $\chi_{\omega}$ be the function defined by

$$
\begin{gathered}
\chi_{\omega}: L^{2}(\Omega) \rightarrow L^{2}(\omega) \\
\quad x \rightarrow \chi_{\omega} x=\left.x\right|_{\omega}(10)
\end{gathered}
$$

where $x \mathrm{I}_{\omega}$ is the restriction of the state $x$ to $\omega$.

- Here, we recall the concept of regional observability as in [12, 21].

Definition 2.1: A system (1) augmented with output function (2) is exactly $\omega$ - observable if:

$$
\operatorname{Im} \chi_{\omega} K^{*}=L^{2}(\omega)
$$

Definition 2.2: A system (1) - (2) is approximately $\omega$ - observable if:

$$
\overline{\operatorname{Im} \chi_{\omega} K^{*}}=L^{2}(\omega)
$$

Definition 2.3: A suite of sensors $\left(D_{i}, g_{i}\right)_{1 \leq i \leq q}$ is $\omega$-strategic if the system (1)-(2) is approximately $\omega$-observable.

The concept of $\omega$ - strategic has been extended to the regional boundary case for $t \in[0, T]$ as in [26, 27] and to the case $t \in(0, \infty)$ as in $[2,10]$. It is said that a suite of $\left(D_{i}, g_{i}\right)_{1 \leq i \leq q}$ is $\omega$-strategic if there exist at least one sensor $\left(D_{1}, g_{1}\right)$ which is $\omega$ - strategic.

Let us consider the orthonormal set $\left(\varphi_{n_{j}}\right)$ of eigenfunctions of $L^{2}(\Omega)$ orthonormal $\operatorname{in} L^{2}(\Omega)$ is associated with eigenvalues $\lambda_{n}$ of multiplicity $r_{n}$ and suppose that the system (1) has $J$ unstable modes. Then, we have the following result.

Proposition 2.4: The sequence of sensors $\left(D_{i}, g_{i}\right)_{1 \leq i \leq q}$ is $\omega$-strategic if and only if

(1) $q \geq r$

(2) $\operatorname{Rank}_{n}=r_{n}$, for all $n, n=1, \ldots, J$ with

$G_{n}=\left(G_{n}\right)_{i_{j}}=\left\{\begin{array}{l}<\varphi_{n_{j}}, g_{i}(.)>_{L^{2}\left(D_{i}\right)} \\ \varphi_{n_{j}}\left(b_{i}\right),\end{array}\right.$

in the zone case

in the pointwise case ${ }^{(11)}$

where $\sup _{n}=r$ and $J=1, \ldots, r_{n}$.

Proof: The proof of this proposition is similar to the rank condition in [12]; the main difference is that the rank condition as follows

$\operatorname{RankG}_{n}=r_{n}, \quad \forall n$

For proposition 2.4, we need only to hold for

$\operatorname{Rank} G_{n}=r_{n}$, for all $n, n=1, \ldots, J$.

\subsection{Definitions and Characterizations}

This section presents some definitions and characterizations related to the regional exponential behaviour.

Definition 2.5: The semi-group $\left(S_{A}(t)\right)_{t \geq 0}$ is said to be exponentially stable if there exist two positive constants $M$ and $\alpha$ such that

$$
\left\|S_{A}(t)\right\|_{X} \leq M e^{-\alpha t} ; t \geq 0
$$

If $\left(S_{A}(t)\right)_{t \geq 0}$ is an exponentially stable semi-group, then for all $x_{0}(.) \in X$, the solution of the associated autonomous system satisfies

$$
\|x(., t)\|_{X}=\left\|S_{A}(t) x_{0}(.)\right\|_{X} \leq M e^{-\alpha t}\left\|x_{0}(.)\right\|_{X}
$$


and therefore

$$
\lim _{t \rightarrow \infty}\|x(., t)\|_{X}=0
$$

we shall think about the next usual definition of stability.

Definition 2.6: The system (1) is said to be stable if the operator $A$ generates a semi-group which is exponentially stable.

Definition 2.7: A semi-group is exponentially regionally stable in $L^{2}(\omega)$ (or $\omega_{E}$-stable) if, for every initial state $x_{0}(.) \in$ $L^{2}(\Omega)$, the solution of the autonomous system associated with (1) converges exponentially to zero when $t \rightarrow \infty$.

Definition 2.8: The system (1) is said to be exponentially stable on $\omega$ (or $\omega_{E}$-stable) if the operator $\mathrm{A}$ generates a semigroup which is $\left(\omega_{E}\right.$ - stable).

It is easy to see that the system (1) is $\omega_{E}$-stable if and only if, for some positive constants $M_{\omega}$ and $\alpha_{\omega}$,

$\left\|\chi_{\omega} S_{A}(t)\right\|_{\mathcal{L}\left(z, L^{2}(\omega)\right)} \leq M_{\omega} e^{-\alpha_{\omega} t} \mathrm{t} \geq 0(14)$

if $\left(S_{A}(t)\right)_{t \leq 0}$ is $\omega_{E}$ - stable, then, for all $x_{0}(.) \in L^{2}(\Omega)$, the solution of autonomous system associated with (1) satisfies

$\|x(., t)\|_{L^{2}(\omega)} \leq M_{\omega} e^{-\alpha_{\omega} t}\left\|x_{0}(.)\right\|_{L^{2}(\omega)}(15)$ and then

$\lim _{t \rightarrow \infty}\|x(., t)\|_{L^{2}(\omega)}=0(16)$

Definition 2.9:The system (1)-(2) is said to be exponentially detectable on $\omega$ (or $\omega_{E}$-detectable) if there exists an operator $H_{\omega}: R^{q} \rightarrow L^{2}(\omega)$ such that $\left(A-H_{\omega} C\right)$ generates a strongly continuous semi-group $\left(S_{H_{\omega}}(t)\right)_{t \leq 0}$ which is $\omega_{E}-$ stable.

\section{REGIONAL EXPONENTIAL GENERAL OBSERVABILITY}

In this section, we shall extend these results as is refer $[3,7]$ to the general regional case by considering $\omega$ as sub-region of $\Omega$. Thus, a new exponential general approach is introduced depending on general estimator in the region $\omega$ may be called regional exponential general observability $\left(\omega_{E_{G}}\right.$-observability). The characterization of this concept needs some assumptions which are related to the exponential behaviour.

\section{1 $\omega_{E_{G}}$ - Estimator Reconstruction Method}

Consider now the system and the output specified by

$$
\left\{\begin{array}{c}
\frac{\partial x}{\partial t}(\xi, t)=A x(\xi, t)+B u(t) \Theta \\
x(\xi, 0)=x_{0}(\xi) \Omega \\
x(\eta, t)=0 \\
z(\xi, t)=C x(\xi, t) \Theta
\end{array}\right.
$$

Let $\omega \subset \Omega$ be a given subdomain (region) of $\Omega$ and assume that for $T \in \mathcal{L}\left(L^{2}(\Omega)\right.$ ), and $T_{\omega}=\chi_{\omega} T$ (where $\chi_{\omega}$ is defined in (10)) there exists a system with state $y(\xi, t)$ such that

$y(\xi, t)=T_{\omega} x(\xi, t)$.

Thus, if we can build a system which is an exponential estimator for $y(\xi, t)$, then it will also be an exponential estimator for $T_{\omega} x(\xi, t)$, that is to say an exponential estimator to the restriction of $T x(\xi, t)$ to the region $\omega$. The equations (2)-(18) give

$\left[\begin{array}{l}Z \\ y\end{array}\right]=\left[\begin{array}{c}C \\ T_{\omega}\end{array}\right] x$

If we assume that there exist two linear bounded operators $R$ and $S$, where $R: \mathcal{O} \rightarrow L^{2}(\omega)$, and $S: L^{2}(\omega) \rightarrow L^{2}(\omega)$, such that $R C+S T_{\omega}=I$, then by deriving $y(\xi, t)$ in $(3.18)$ we have 


$$
\begin{aligned}
\frac{\partial y}{\partial t}(\xi, t) & =T_{\omega} \frac{\partial x}{\partial t}(\xi, t)=T_{\omega} A x(\xi, t)+T_{\omega} B u(t) \\
& =T_{\omega} A S y(\xi, t)+T_{\omega} A R z(\xi, t)+T_{\omega} B u(t)
\end{aligned}
$$

Consider now the system (which is destined to be the regional exponential general estimator)

$$
\begin{cases}\frac{\partial \hat{y}}{\partial t}(\xi, t)=F_{\omega} \hat{y}(\xi, t)+G_{\omega} u(\xi, t)+\mathcal{H}_{\omega} z(\xi, t) \Theta & \Omega \\ \hat{y}(\xi, 0)=\hat{y}_{0}(\xi) & \Pi \\ \hat{y}(\eta, t)=0 & \Pi\end{cases}
$$

where $F_{\omega}$ generates a strongly continuous semi-group $\left(S_{F_{\omega}}(t)\right)_{t \geq 0}$, which is regionally exponentially stable on $X=L^{2}(\omega)$, i.e.,

$\exists M_{F_{\omega}}, \alpha_{F_{\omega}}>0$, suchthat $\left\|S_{F_{\omega}}(.)\right\|_{L^{2}(\omega)} \leq M_{F_{\omega}} e^{-\alpha_{F_{\omega}} t} \forall t \geq 0(21)$

and $G_{\omega} \in \mathcal{L}\left(U, L^{2}(\omega)\right)$ and $\mathcal{H}_{\omega} \in \mathcal{L}\left(\mathcal{O}, L^{2}(\omega)\right)$. The solution of (20) is given by

$\hat{y}(\xi, t)=S_{F_{\omega}}(t) \hat{y}_{0}(\xi)+\int_{0}^{t} S_{F_{\omega}}(t-\tau)\left[G_{\omega} u(t)+\mathcal{H}_{\omega} z(\xi, \tau)\right] d \tau$

Now, we want to show that under convenient hypothesis, the state of the system (20) is a regional exponential general estimator of $T_{\omega} x(\xi, t)$ (Figure 3). For this purpose we need to decompose the system (1) as in next subsection.

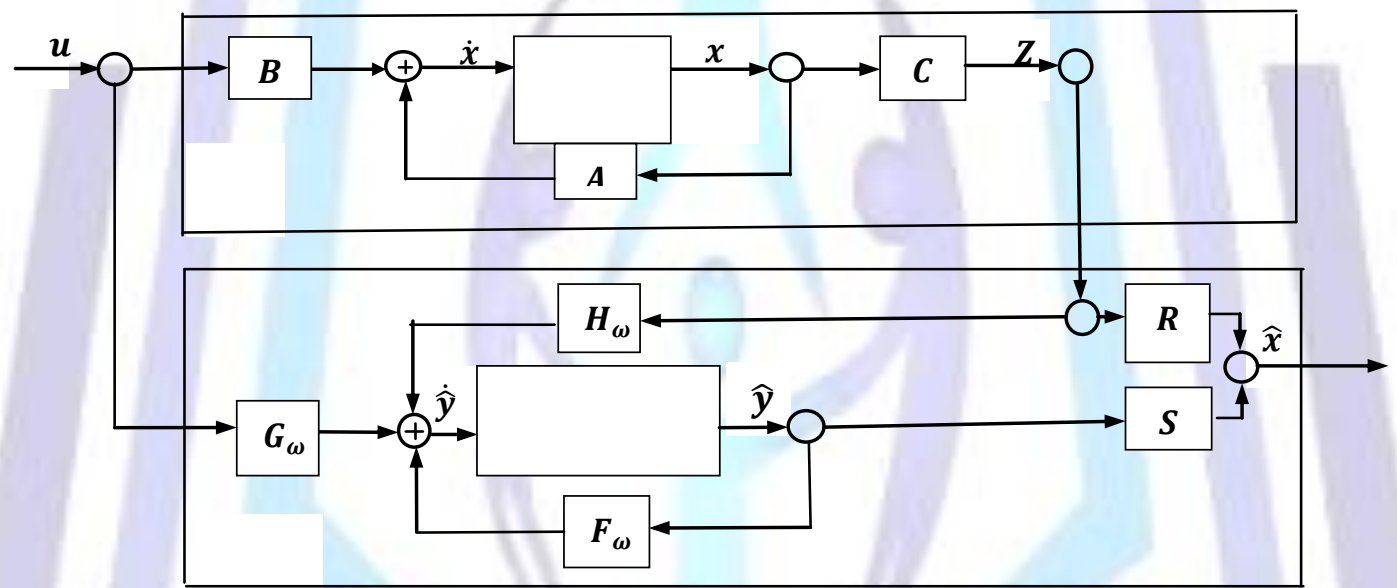

Fig. 3: Regional exponential general observability.

\subsection{General Decomposed System}

Now, under the assumption of strongly continuous semi-group we have the system (1)-(2) is reduced as in the additional assumptions allow a decomposition of (1) to a form of the stabilizing operator $\mathcal{H}$. These assumptions are as follows.

(1) $A$ has a pure point spectrum, denoted by $\sigma(A)$.

(2) $S_{A}(t)$ is a compact operator for some $t>0$

(3) For $\delta>0, \sigma(A)$ the spectrum of $A$ contained in the closed half plane $\{\lambda: \operatorname{Re} \lambda \geq-\delta\}$.

(4) The subspace associated with each finite dimensional point of $\sigma(A)$ in the half plane $\{\lambda: \operatorname{Re} \lambda \geq-\delta\}$.

(5) Ois finite dimensional.

These five assumptions are strong. The Hille-Yosida theorem implies that the set of spectral point of $A$ lying in the half plane $\{\lambda: \operatorname{Re} \lambda \geq-\delta\}$ forms a bounded spectral set [25]. Denote this spectral set by $\sigma\left(A_{1}\right)$. Using the spectral set $\sigma\left(A_{1}\right)$, a reduced form of (1) can be derived. Denote $\sigma(A)-\sigma\left(A_{1}\right)$ by $\sigma\left(A_{2}\right)$. As $A$ is a closed operator with nonempty resolvent set, operational calculus can be used to completely reduce the operator $A$ in terms of the spectral sets $\sigma\left(A_{1}\right)$ and $\sigma\left(A_{2}\right)$ [15]. $\sigma\left(A_{1}\right)$ and $\sigma\left(A_{2}\right)$ determine subspaces $X_{1}$ and $X_{2}$,

$X=X_{1} \oplus X_{2}$,

and projections $E_{1}: X \rightarrow X_{1}, E_{2}: X \rightarrow X_{2}$, such that

$$
\begin{aligned}
& E_{1} A x=A E_{1} x \\
& E_{2} A x=A E_{2} x
\end{aligned}
$$


Defining $A_{1} x=A E_{1} x, \quad D\left(A_{1}\right)=D(A) \cap X_{1}$

and $A_{2} x=A E_{2} x, D\left(A_{2}\right)=D(A) \cap X_{2}$

the operator $A$ can be represented by

$A x=\left[\begin{array}{cc}A_{1} & 0 \\ 0 & A_{2}\end{array}\right]\left[\begin{array}{l}x_{1} \\ x_{2}\end{array}\right]$ and $B=\left[\begin{array}{l}B_{1} \\ B_{2}\end{array}\right]$

Where $x=x_{1}+x_{2}$,

$x \in D(A), x_{1} \in D\left(A_{1}\right), x_{2} \in D\left(A_{2}\right), B_{1} \in \mathcal{L}\left(R^{P}, X_{1}\right)$ and $B_{2} \in \mathcal{L}\left(R^{P}, X_{2}\right)$

as $D(A)$ is dense in $X, D\left(A_{1}\right)$ is dense in $X_{1}$, and $D\left(A_{2}\right)$ is dense in $X_{2}$.

$A_{1}$ and $A_{2}$ are closed operators as $A$ is closed operator. If $A$ is the infinitesimal generator of a strongly continuous semigroup, then the Hille-Yosida theorem shows that both $A_{1}$ and $A_{2}$ are infinitesimal generators. Using the decomposition of $X$ and $A$ given by (23)-(24), and then (1)-(2) can be rewritten in the following forms [7]

$\begin{cases}\frac{\partial x_{1}}{\partial t}(\xi, t)=A_{1} x_{1}(\xi, t)+E_{1} B_{1} u(t) & \Theta \\ x_{1}(\xi, 0)=x_{1_{0}}(\xi) & \Omega \\ x_{1}(\eta, t)=0 & \Pi\end{cases}$
and
$\begin{cases}\frac{\partial x_{2}}{\partial t}(\xi, t)=A_{2} x_{2}(\xi, t)+E_{2} B_{2} u(t) & \Theta \\ x_{2}(\xi, 0)=x_{2_{0}}(\xi) & \Omega \\ x_{2}(\eta, t)=0 & \Pi\end{cases}$

Augmented with the output function

$y(., t)=C x_{1}(\xi, t)(27)$

Equations (26)-(27) are called the reduced form of (1)-(2).

Since $A_{1}$ is the restriction of $A$ to $X_{1}$, and $D\left(A_{1}\right)=D(A) \cap X_{1}$, the spectrum of $A_{1}$ is $\sigma\left(A_{1}\right)$ [25]. As the points of $\sigma\left(A_{1}\right)$ are isolated, each point by itself is a spectral set, and the spectral sets so formed are pairwise disjoint. Thus a projection $E_{i j}$ and subspace $X_{i j}$ can be associated with each point $\lambda_{j} \in \sigma\left(A_{1}\right)$, and the subspace $X_{1}$ completely reduced to

$$
X_{1}=X_{11} \oplus X_{12} \oplus \ldots \oplus X_{\text {in }}
$$

wheren is the number of points in $\sigma\left(A_{1}\right)$. Each $X_{i j}$ is finite dimensional by assumption, hence $X_{1}$ is finite dimensional, and $A_{1}$ is a bounded operator. Then choosing bases for $X_{1}$ and $\mathcal{O},(26)$ can be represented as a linear constant coefficient ordinary differential equation, and $C$ restricted to $X_{1}$ can be expressed as a matrix.

In terms of the finite dimensional bases for $X_{1}$ and $\mathcal{O}$, the homogeneous equations corresponding to (26)-(27) are

$\left\{\begin{array}{c}\frac{\partial x_{1}}{\partial t}(\xi, t)=A_{1} x_{1}(\xi, t) \\ x_{1}(\xi, 0)=x_{1_{0}}(\xi)\end{array}\right.$

$\left\{\begin{array}{c}\frac{\partial x_{2}}{\partial t}(\xi, t)=A_{2} x_{2}(\xi, t) \\ x_{2}(\xi, 0)=x_{2_{0}}(\xi)\end{array}\right.$

$y(., t)=C x_{1}(\xi, t)$

Where $\Gamma$ is the coordinate space associated with the basis for $X_{1}$, and $C: X_{1} \rightarrow \mathcal{O}$ in terms of the bases of $X_{1}$ and $\mathcal{O}$.

An estimate will now be made of the solutions of (29). A having a pure point spectrum implies that $A_{2}$ has a pure point spectrum, while $S_{A_{2}}(t)$ being a compact operator for some $t>0$ implies that $S_{A_{2}}(t)$ is a compact operator. As $S_{A_{2}}(t)$ is a compact operator, its spectrum consists of only point spectrum, denoted by $\mathcal{P} \sigma\left(S_{A_{2}}(t)\right)$ is given by

$e^{\mathcal{P} \sigma\left(A_{2}\right) t}$, as $\operatorname{Re} \sigma\left(A_{2}\right) \leq-\delta, \quad\left\|e^{\mathcal{P} \sigma\left(A_{2}\right) t}\right\| \leq e^{-\delta t}$

Then the spectral radius of $S_{A_{2}}(t)$ satisfies

$r_{\sigma}\left(S_{A_{2}}(t)\right) \leq e^{-\delta t}$.

using a lemma of Hale [16]. For any $\gamma>0$ there exists an $M(\gamma) \geq 1$ such that 


$$
\left\|S_{A_{2}}(t) x_{2_{0}}\right\| \leq M\left(x_{1}\right) e^{\left(-\delta+x_{1}\right) t}\left\|x_{2_{0}}\right\|
$$

for all $t \geq 0$ and $x_{2_{0}} \in X_{2}$. Thus, (29) is exponentially stable.

\section{3 $\omega_{E_{G}}-$ Observability and $\omega_{E_{G}}$ - Detectability}

This subsection, explores the relation between regional exponential general observability and regional exponential general detectability in order to characterize the concept of regional exponential general observability.

Here, we introduce some definitions and characterization concern to the concept of regional exponential general observability.

Definition 3.1: Suppose that there exists a dynamical system with state $y(\xi, t) \in Y$ (a Hilbert space) given by

$$
\left\{\begin{array}{l}
\frac{\partial y}{\partial t}(\xi, t)=F_{\omega} y(\xi, t)+G_{\omega} u(t)+\mathcal{H}_{\omega} z(\xi, t) \\
y(\eta, t)=0
\end{array} \quad \begin{array}{l}
\Theta(\xi, 0)=y_{0}(\xi) \Omega \\
\Pi
\end{array}\right.
$$

where $F_{\omega}$ generates a strongly continuous semi-group $\left(S_{F_{\omega}}(t)\right)_{t \leq 0}$ which is stable on Hilbert space $\mathrm{Y}, G_{\omega} \in \mathcal{L}(U, Y)$ and $\mathcal{H}_{\omega} \in \mathcal{L}(\mathcal{O}, Y)$. The system $(31)$ defines an $\omega_{E_{G}}$ - estimator for $\chi_{\omega} T x(\xi, t)$ if

(1) $\lim _{t \rightarrow \infty}\left\|\chi_{\omega} T x(\xi, t)-y(\xi, t)\right\|_{L^{2}(\omega)}=0$

(2) $\chi_{\omega} T$ maps $D(A)$ in $D\left(F_{\omega}\right)$ where $x(\xi, t)$ and $y(\xi, t)$ are the solution of the system (1) and (31).

Definition 3.2: The system (1)-(2) is regional exponential general observable in $\omega\left(\omega_{E_{G}}\right.$-observable) if there exists a dynamical system (31) $\left(\omega_{E_{G}}\right.$ - estimator) for the original system.

Now, we can extend these results to the case of exponential general detectability:

Definition 3.3: The system (1) is said to be exponential general stable on $\omega$ (or $\omega_{E_{G}}$-stable) if the operator $T_{\omega} A$ generates a semi-group which is $\left(\omega_{E_{G}}\right.$-stable). It is easy to see that the system (1) is $\omega_{E_{G}}$-stable if and only if, for some positive constants $M_{\omega}$ and $\alpha_{\omega}$,

$\left\|S_{T_{\omega} A}(.)\right\|_{\mathcal{L}\left(Z, L^{2}(\omega)\right)} \leq M_{\omega} e^{-\alpha_{\omega} t} t \geq 0$

If $\left(S_{T_{\omega} A}(t)\right)_{t \geq 0}$ is $\omega_{E_{G}}$-stable, then, for all $x_{0}(.) \in L^{2}(\Omega)$, the solution of autonomous system associated with (1) satisfies:

$\|x(t)\|_{L^{2}(\omega)}=\left\|S_{T_{\omega} A}(.) x_{0}\right\|_{L^{2}(\omega)} \leq M_{\omega} e^{-\alpha_{\omega} t}\left\|x_{0}\right\|_{L^{2}(\omega)}(33)$

and then

$\lim _{t \rightarrow \infty}\|x(t)\|_{L^{2}(\omega)}=0$

Definition 3.4: The system (1)-(2) is said to be exponential general detectable on $\omega$ (or $\omega_{E_{G}}$-detectable) if there exists an operator $T_{\omega} F_{\omega}: R^{q} \rightarrow L^{2}(\omega)$ such that $\left(T_{\omega} A-\mathcal{H}_{\omega} C\right)$ generates a strongly continuous semi-group $\left(S_{T_{\omega} F_{\omega}}(t)\right)_{t \leq 0}$ which is $\omega_{E_{G}}-$ stable.

We can extend definitions 2.1, 2.2 and 2.3 to case of general observability for general decomposed systems:

Definition 3.5: A system (1) - (2) is exactly $\omega_{G}$ - observable if:

$$
\operatorname{Im} \chi_{\omega} K^{*}=X_{1}
$$

Definition 3.6: A system (1)-(2) is approximately $\omega_{G}$ - observable if:

$$
\overline{\operatorname{Im} \chi_{\omega} K^{*}}=X_{1}
$$


Definition 3.7: A suite of sensors $\left(D_{i}, g_{i}\right)_{1 \leq i \leq q}$ is $\omega_{G}$-strategic if the system (1)-(2) is approximately $\omega_{G}$-observable.

Now, the approach which observes exponentially the current state $x(\xi, t)$ in a region $\omega$ is given by the following result:

Theorem 3.8:Suppose that there are $q$ sensors $\left(D_{i}, f_{i}\right)_{1 \leq i \leq q}$ and the spectrum of $T_{\omega} A$ contains $J$ eigenvalues with nonnegative real parts. The system (1)-(2) is $\omega_{E_{G}}$-detectable iff

1. $q \geq m$

2. Rank $G_{i}=m_{i}, \forall i, i=1, \ldots, J$ with

$$
G=G_{i j}= \begin{cases}\left\langle\varphi_{j}(.), g(.)\right\rangle_{L^{2}\left(D_{i}\right)}, & \text { for zone sensors } \\ \varphi_{j}\left(b_{i}\right), & \text { for pointwise sensors } \\ \left\langle\varphi_{j}(.), g(.)\right\rangle_{L^{2}\left(\Gamma_{i}\right)}, & \text { for boundary zone sensors }\end{cases}
$$

wheresup $m_{i}=m<\infty$ and $j=1, \ldots, \infty$.

Proof: The proof is limited to the case of zone sensors.

1. Under the hypothesis of the problem statement in (Section 3.1), the system (1) can be decomposed by the projections $\mathcal{P}$ and $I-\mathcal{P}$ on two parts, unstable and stable under the assumptions of (25)-(27), where $\mathcal{P}$ and $I-\mathcal{P}$ play the role of projection as $E_{1}, E_{2}$ in section 3.2. The state vector may be given by $x(\xi, t)=\left[x_{1}(\xi, t)+x_{2}(\xi, t)\right]^{t_{r}}$ where $x_{1}(\xi, t)$ is the state component of the unstable part of the system (1) and may be written in the form

$\left\{\begin{array}{c}\frac{\partial x_{1}}{\partial t}(\xi, t)=T_{\omega} A_{1} x_{1}(\xi, t)+\mathcal{P} B u(t) \Theta \\ x_{1}(\xi, 0)=x_{0_{1}}(\xi) \Omega \\ x_{1}(\eta, t)=0\end{array}\right.$

and $x_{2}(\xi, t)$ is the component state of the part of the system (1) given by

$\left\{\begin{array}{c}\frac{\partial x_{2}}{\partial t}(\xi, t)=T_{\omega} A_{2} x_{2}(\xi, t)+(I-\mathcal{P}) B u(t) \Theta \\ \\ x_{2}(\xi, 0)=x_{0_{2}}(\xi) \Omega \\ x_{2}(\eta, t)=0\end{array}\right.$

the operator $T_{\omega} A_{1}$ is represented by matrix of order $\left(\sum_{i=1}^{J} m_{i}, \sum_{i=1}^{J} m_{i}\right)$ given by

$T_{\omega} A_{1}=\operatorname{diag}\left[\lambda_{1}, \ldots, \lambda_{1}, \lambda_{2}, \ldots, \lambda_{2}, \ldots, \lambda_{J}, \ldots, \lambda_{J}\right]$

$$
\mathcal{P} B=\left[G_{1}^{t_{r}}, G_{2}^{t_{r}}, \ldots, G_{J}^{t_{r}}\right]
$$

By using the condition (2) of this theorem, we deduce that the suite $\left(D_{i}, g_{i}\right)_{1 \leq i \leq q}$ of sensors is $\omega_{G}$-strategic for the unstable part of the system (1). The subsystem (35) is approximately $\omega_{G}$-observable [7], and since it is finite dimensional, then it is exactly $\omega_{G}$-observable [20]. Therefore, it is $\omega_{E_{G}}$-detectable, and hence there exists an operator $\mathcal{H}_{\omega}^{1}$ such that $T_{\omega} A_{1}-\mathcal{H}_{\omega}^{1} C$ which satisfies the following: $\exists M_{\omega}^{1}, \alpha_{\omega}^{1}>0$

such that $\left\|e^{\left(T_{\omega} A_{1}-\mathcal{H}_{\omega}^{1} C\right) t}\right\| \leq M_{\omega}^{1} e^{-\alpha_{\omega}^{1} t}$ and, then, we have

$\left\|x_{1}(., t)\right\|_{L^{2}(\omega)} \leq M_{\omega}^{1} e^{-\alpha_{\omega}^{1} t}\left\|P x_{0}\right\|_{L^{2}(\omega)}$

Since the semi-group generated by the operator $T_{\omega} A_{2}$ is $\omega_{E_{G}}$-stable, there exists $M_{\omega}^{2}, \alpha_{\omega}^{2}>0$ such that

$$
\begin{aligned}
& \left\|x_{2}(., t)\right\|_{L^{2}(\omega)} \leq M_{\omega}^{1} e^{-\alpha_{\omega}^{1} t}\left\|(I-P) x_{0_{2}}(.)\right\|_{L^{2}(\omega)} \\
& +\int_{0}^{t} M_{\omega}^{2} e^{-\alpha_{\omega}^{2} t}\left\|(I-P) x_{0_{2}}(.)\right\|_{L^{2}(\omega)}\|u(\tau)\| d \tau
\end{aligned}
$$

and therefore $\left\|x_{1}(\xi, t)\right\|_{L^{2}(\omega)} \rightarrow 0$ when $t \rightarrow \infty$. Finally, the system (1)-(2) is $\omega_{E_{G}}$-detectable.

2. If the system (1)-(2) is $\omega_{E_{G}}$-detectable, then

$\exists \mathcal{H}_{\omega} \mathcal{L}\left(L^{2}\left(0, \infty, R^{q}\right), L^{2}(\omega)\right)$ such that $\left(T_{\omega} A-\mathcal{H}_{\omega} C\right)$ generates an $\omega_{E_{G}}$-stable, strongly continuous semi-group

$\left(S_{T_{\omega} F_{\omega}}(t)\right)_{t \geq 0}$ on the space $L^{2}(\omega)$ which satisfies the follwing

$\exists M_{\omega}, \alpha_{\omega}>0$ such that 


$$
\left\|S_{T_{\omega} F_{\omega}}(t)\right\|_{L^{2}(\omega)} \leq M_{\omega} e^{-\alpha_{\omega}(t)}
$$

Thus the unstable subsystem (35) is $\omega_{E_{G}}$-detectable. Since this subsystem is of finite dimensional, then it is exactly $\omega_{G}$-observable. Therefore (35) is approximately $\omega_{G}$-observable and hence it is reduced $\omega_{G}$-strategic, i.e.,

we have

$$
\left[\mathcal{K} \chi_{\omega}^{*} x^{*}(., t)=0 \Rightarrow x^{*}(., t)=0\right] \text {. For } x^{*}(., t) \in L^{2}(\omega)
$$

$$
\mathcal{K} \chi_{\omega}^{*} x^{*}(., t)=\left(\sum_{j=1}^{J} e^{\lambda_{j} t}\left\langle\varphi_{j}(.), x^{*}(., t)\right\rangle_{L^{2}(\omega)}\left\langle\varphi_{j}(.), g_{i}(.)\right\rangle_{L^{2}(\Omega)}\right)_{1 \leq i \leq q}
$$

If the unstable system (35) is not $\omega_{G}$-strategic, $\exists x^{*}(., t) \in L^{2}(\omega)$ such that $\mathcal{K} \chi_{\omega}^{*} x^{*}(., t)=0$ this leads to

$$
\sum_{j=1}^{J}\left\langle\varphi_{j}(.), x^{*}(., t)\right\rangle_{L^{2}(\omega)}\left\langle\varphi_{j}(.), g(.)\right\rangle_{L^{2}(\Omega)}=0
$$

the state vectors $x_{i}$ may be given by

$$
x_{i}(., t)=\left[\left\langle\varphi_{1}(.), x^{*}(., t)\right\rangle_{L^{2}(\omega)}\left\langle\varphi_{j}(.), x^{*}(., t)\right\rangle_{L^{2}(\omega)}\right]^{t r} \neq 0
$$

we then obtain $G_{i} x_{i}=0, \forall i, i=1, \ldots$, Jand therefore $\operatorname{Rank} G_{i} \neq m_{i}$

Now, we present the sufficient condition of regional exponential general observability concept in the following result.

Theorem 3.9: The system (17) is $\omega_{E_{G}}$-observable by the dynamical system (20), If the following conditions hold:

(1) There exist $R \in \mathcal{L}\left(\mathcal{O}, L^{2}(\omega)\right)$ and $S \in \mathcal{L}\left(L^{2}(\omega)\right)$ such that

$R C+S T_{\omega}=I$

(2) $\left\{\begin{array}{c}T_{\omega} A-F_{\omega} T_{\omega}=\mathcal{H}_{\omega} C \\ \text { and } G_{\omega}=T_{\omega} B\end{array}\right.$

Proof: For $y(\xi, t)=T_{\omega} x(\xi, t)$ and $\hat{y}(\xi, t)$ solution of $(20)$, denote $\phi(\xi, t)=y(\xi, t)-\hat{y}(\xi, t)$. We have

$$
\begin{gathered}
\frac{\partial \phi}{\partial t}(\xi, t)=\frac{\partial y}{\partial t}(\xi, t)-\frac{\partial \hat{y}}{\partial t}(\xi, t) \\
=T_{\omega} A x(\xi, t)+T_{\omega} B u(t) \\
-F_{\omega} \hat{y}(\xi, t)-G_{\omega} u(t)-\mathcal{H}_{\omega} z(\xi, t) \\
=F_{\omega} \phi(\xi, t)-F_{\omega} y(\xi, t)+T_{\omega} A x(\xi, t) \\
-\mathcal{H}_{\omega} z(\xi, t)+T_{\omega} B u(t)-G_{\omega} u(t) \\
=F_{\omega} \phi(\xi, t)+\left[T_{\omega} A x(\xi, t)-F_{\omega} T_{\omega} x(\xi, t)\right.
\end{gathered}
$$

$$
\begin{aligned}
& \left.-\mathcal{H}_{\omega} C x(\xi, t)\right]+\left[T_{\omega} B-G_{\omega}\right] u(t) \\
& =F_{\omega} \phi(\xi, t)+\left[T_{\omega} A-F_{\omega} T_{\omega}-\mathcal{H}_{\omega} C\right] x(\xi, t) \\
& \quad+\left[T_{\omega} B-G_{\omega}\right] u(t)=F_{\omega} \phi(\xi, t)
\end{aligned}
$$

Consequently, $\phi(\xi, t)=S_{F_{\omega}}(t) \phi(0, t)$ where $\phi_{0}(t)=\phi(0, t)=T_{\omega} x_{0}(\xi)-\hat{y}_{0}(\xi)$. Since the operator $F_{\omega} T_{\omega}$ generates a strongly continuous semi-group $\left(S_{T_{\omega} F_{\omega}}(t)\right)_{t \leq 0}$ which is $\omega_{E_{G}}$-stable, thenby using equation (21) we obtain

$$
\|\phi(\xi, t)\|_{L^{2}(\omega)} \leq M_{F} e^{-\alpha_{F} t}\left\|\phi_{0}(t)\right\|_{L^{2}(\omega)},
$$

and therefore $\lim _{t \rightarrow \infty} \phi(\xi, t)=0$.

Consider now $\hat{x}(\xi, t)=R z(\xi, t)+S \hat{y}(\xi, t)$, we have

$$
\begin{aligned}
& \hat{x}(\xi, t)=x(\xi, t)-\hat{x}(\xi, t) \\
& =x(\xi, t)-R z(\xi, t)-S \hat{y}(\xi, t) \\
& =x(\xi, t)-R C x(\xi, t)-S T_{\omega} x(\xi, t)
\end{aligned}
$$




$$
\begin{aligned}
& +S\left[T_{\omega} x(\xi, t)-\hat{y}(\xi, t)\right] \\
= & S\left[T_{\omega} x(\xi, t)-\hat{y}(\xi, t)\right. \\
= & S[y(\xi, t)-\hat{y}(\xi, t)] \\
= & S \phi(\xi, t) .
\end{aligned}
$$

Consequently we have

$\lim _{t \rightarrow \infty}\|\hat{x}(\xi, t)-x(\xi, t)\|_{L^{2}(\omega)}=0$.

Thus, we have

$$
\lim _{t \rightarrow \infty}\left\|T_{\omega} x(\xi, t)-\hat{y}(\xi, t)\right\|_{L^{2}(\omega)}=0
$$

and the dynamical system (31) is $\omega_{E_{G}}$-estimator. Finally the system (17) is $\omega_{E_{G}}$ - observable.

From the previous theorem 3.9 we can deduce that:

- Conditions (40) and (41) in theorem 3.9 guarantee that the dynamical system (1)-(2) is $\omega_{E_{G}}$ - observable.

- A system which is $\Omega_{E_{G}}$-observable, it is $\omega_{E_{G}}$-observable

- If a system is $\omega_{E_{G}}$ - observable in $\omega$, then it is $\omega_{E_{G}}^{1}$ - observable every subset $\omega^{1} \subset \omega$, but the converse is not true.

This is illustrated in the following counter example.

Counter Example 3.10: Consider the case of two dimensional distributed parameter diffusion system defined in $\Omega=(0,1) \times$ $(0,1)$ and described by the parabolic question

$\left\{\begin{array}{c}\frac{\partial x}{\partial t}\left(\xi_{1}, \xi_{2}, t\right)=\frac{\partial^{2} x}{\partial \xi_{1}^{2}}\left(\xi_{1}, \xi_{2}, t\right)+\frac{\partial^{2} x}{\partial \xi_{2}^{2}}\left(\xi_{1}, \xi_{2}, t\right)+x\left(\xi_{1}, \xi_{2}, t\right) \Theta \\ x\left(\xi_{1}, \xi_{2}, 0\right)=x_{0}\left(\xi_{1}, \xi_{2}\right) \Omega \\ x\left(\eta_{1}, \eta_{2}, t\right)=0\end{array}\right.$

with the output function

$z(t)=\int_{\Gamma} \frac{\partial x}{\partial v}\left(\eta_{1}, \eta_{2}, t\right) g\left(\eta_{1}, \eta_{2}\right) d \xi_{1} d \xi_{2}$

The above system represents the heat-conduction problem. The measurement is given via zone $\operatorname{sensor}(\Gamma, g)$ defined by $\Gamma=\left[\eta_{0_{1}}-I_{1}, 1\right] \times\{0\} \cup\{1\} \times\left[0, \eta_{0_{2}}+I_{2}\right] \subset \partial \Omega$. The operator $A=\left(\frac{\partial^{2}}{\partial \xi_{1}^{2}}+\frac{\partial^{2}}{\partial \xi_{2}^{2}}+1\right)$ generates a strongly continuous semigroup $\left(S_{A}(t)\right)_{t \geq 0}$ on the Hilbert space $L^{2}(\Omega)$. Consider the dynamical system

$\left\{\begin{array}{c}\frac{\partial y}{\partial t}\left(\xi_{1}, \xi_{2}, t\right)=\frac{\partial^{2} y}{\partial \xi_{1}^{2}}\left(\xi_{1}, \xi_{2}, t\right)+\frac{\partial^{2} y}{\partial \xi_{2}^{2}}\left(\xi_{1}, \xi_{2}, t\right)+y\left(\xi_{1}, \xi_{2}, t\right) \Theta \\ +\mathcal{H}\left(z(t)-C y\left(\xi_{1}, \xi_{2}, t\right)\right) \\ y\left(\xi_{1}, \xi_{2}, 0\right)=y_{0}\left(\xi_{1}, \xi_{2}\right) \Omega \\ y\left(\eta_{1}, \eta_{2}, t\right)=0\end{array}\right.$

where $\mathcal{H} \in \mathcal{L}\left(\mathcal{O}, L^{2}(\Omega)\right)$ and $C: L^{2}(\bar{\Omega}) \rightarrow \mathcal{O}$ is a linear operator. If $n\left(\eta_{0_{1}}-\alpha_{1}\right) /\left(\beta_{1}-\alpha_{1}\right)$ and $m\left(\eta_{0_{2}}-\alpha_{2}\right) /\left(\beta_{2}-\alpha_{2}\right) \in \mathbb{N}$ for $n, m \in\{1, \ldots, J\}$, then the sensor $(\Gamma, g)$ is not strategic for unstable subsystem of (43) and therefore the system (42)-(43) is not detectable in $\Omega[19]$. Then, the dynamical systems (44) is not observed for the system (42)-(43) [20]. Here, we consider the region $\omega=\left(\alpha_{1}, \beta_{1}\right) \times\left(\alpha_{2}, \beta_{2}\right) \subset(0,1) \times(0,1)$ (Figure 4$)$ and the dynamic system:

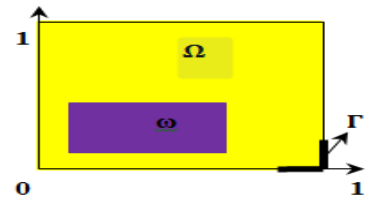

Fig. 4: Domain $\Omega$, region $\omega$, and location $\Gamma$ of the boundary zone sensor. 
$\left\{\begin{array}{cc}\frac{\partial y}{\partial t}\left(\xi_{1}, \xi_{2}, t\right)= & \frac{\partial^{2} y}{\partial \xi_{1}^{2}}\left(\xi_{1}, \xi_{2}, t\right)+\frac{\partial^{2} y}{\partial \xi_{2}^{2}}\left(\xi_{1}, \xi_{2}, t\right)+y\left(\xi_{1}, \xi_{2}, t\right) \Theta \\ & +\mathcal{H}_{\omega}\left(z(t)-C y\left(\xi_{1}, \xi_{2}, t\right)\right) \\ & y\left(\xi_{1}, \xi_{2}, 0\right)=y_{0}\left(\xi_{1}, \xi_{2}\right) \Omega \\ y\left(\eta_{1}, \eta_{2}, t\right)=0 & \end{array}\right.$

where $\mathcal{H}_{\omega} \in \mathcal{L}\left(\mathcal{O}, L^{2}(\omega)\right)$. If $\left(\eta_{0_{1}}-\alpha_{1}\right) /\left(\beta_{1}-\alpha_{1}\right)$ and $m\left(\eta_{0_{2}}-\alpha_{2}\right) /\left(\beta_{2}-\alpha_{2}\right) \in \mathbb{N}$ for everyn, $m=1, \ldots, J$, then the sensor $(\Gamma, g)$ is $\omega$-strategic for the unstable subsystem of (42) [6] and therefore the system (42)-(43) is $\omega$ - detectable, that means $\lim _{t \rightarrow \infty}\|x(\xi, t)-y(\xi, t)\|_{L^{2}(\omega)}=0$ [9]. Finally, the dynamical system (45) is $\omega$ - estimator for the system (42)-(43).

\section{APPLICATION TO TWO DIMENSTIONAL DIFFUSION SYSTEM}

In this section, we consider two-dimensional system defined on $\Omega=(0,1) \times(0,1)$ by the form

$\begin{cases}\frac{\partial x}{\partial t}\left(\xi_{1}, \xi_{2}, t\right)=\frac{\partial^{2} x}{\partial \xi_{1}^{2}}\left(\xi_{1}, \xi_{2}, t\right)+\frac{\partial^{2} x}{\partial \xi_{2}^{2}}\left(\xi_{1}, \xi_{2}, t\right)+x\left(\xi_{1}, \xi_{2}, t\right) & \Theta \\ x\left(\eta_{1}, \eta_{2}, t\right)=0 & x\left(\xi_{1}, \xi_{2}, t\right)=x_{0}\left(\xi_{1}, \xi_{2}\right) \Pi\end{cases}$

together with output function by (4), (5). Let $\omega=\left(\alpha_{1}, \beta_{1}\right) \times\left(\alpha_{2}, \beta_{2}\right)$ be the considered region which is subset of $(0,1) \times$ $(0,1)$. In this case, the eigenfunctions of system $(46)$ are given by

$\varphi_{i j}\left(\xi_{1}, \xi_{2}\right)=\frac{2}{\sqrt{\left(\beta_{1}-\alpha_{1}\right)\left(\beta_{2}-\alpha_{2}\right)}} \sin i \pi\left(\frac{\xi_{1}-\alpha_{1}}{\beta_{1}-\alpha_{1}}\right) \sin j \pi\left(\frac{\xi_{2}-\alpha_{2}}{\beta_{2}-\alpha_{2}}\right)$

associated with eigenvalues

$\lambda_{i j}=-\left(\frac{i^{2}}{\left(\beta_{1}-\alpha_{1}\right)^{2}}+\frac{j^{2}}{\left(\beta_{2}-\alpha_{2}\right)^{2}}\right)$.

The following results give information on the location of internal zone or pointwise regional strategic sensors.

\subsection{Internal Zone Sensor}

Consider the system (46) together with output function (2) where the sensor supports $D_{i}$ are located in $\Omega$ (or $\partial \Omega$ ). The output (2) can be written by the form

$z(t)=\int_{D_{i}} x\left(\xi_{1}, \xi_{2}, t\right) g\left(\xi_{1}, \xi_{2}\right) d \xi_{1} d \xi_{2}$

where $D_{i} \subset \Omega$ is the location of zone sensor and $g \in L^{2}\left(D_{i}\right)$. In this case of (Figure 5 ), the eigenfunctions and the eigenvalues are given by (47)-(48). However, if we suppose that

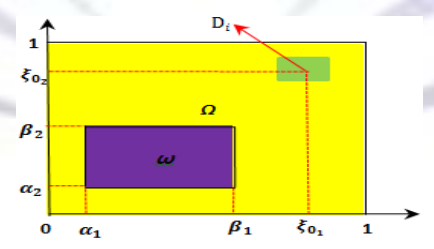

Fig. 5: Domain $\Omega$, subregion $\omega$, and location $D_{i}$ of internal zone sensor.

$\frac{\left(\beta_{1}-\alpha_{1}\right)^{2}}{\left(\beta_{2}-\alpha_{2}\right)^{2}} \notin Q$

then $r=1$ and one sensor may be sufficient to achieve $\omega_{E_{G}}$ - observability [18]. In this case, the dynamical system (28) is given by

$\left\{\begin{array}{rc}\frac{\partial y}{\partial t}\left(\xi_{1}, \xi_{2}, t\right)=\Delta y\left(\xi_{1}, \xi_{2}, t\right)+y\left(\xi_{1}, \xi_{2}, t\right) \Theta \\ +\mathcal{H}_{\omega}\left(<x(\xi, t), g_{i}(\xi)>-C y(\xi, t)\right) \\ y\left(\eta_{1}, \eta_{2}, t\right)=0 & y\left(\xi_{1}, \xi_{2}, 0\right)=y_{0}\left(\xi_{1}, \xi_{2}\right) \Omega\end{array}\right.$ 
the measurement support is rectangular with

$D_{i}=\left[\xi_{1}-I_{1}, \xi_{1}+I_{1}\right] \times\left[\xi_{2}-I_{2}, \xi_{2}+I_{2}\right] \in \Omega$

we then have the result.

Corollary 4.1: If $g_{1}$ is symmetric about $\xi_{1}=\xi_{0_{1}}$ and $g_{2}$ is symmetric about $\xi_{2}=\xi_{0_{2}}$, then the system (46)-(49) is $\omega_{E_{G}}$ observable by the dynamical system $(51)$ if

$\frac{i\left(\xi_{0_{1}}-\alpha_{1}\right)}{\left(\beta_{1}-\alpha_{1}\right)}$ and $\frac{i\left(\xi_{0_{2}}-\alpha_{2}\right)}{\left(\beta_{2}-\alpha_{2}\right)} \notin N$ for some $i=1,2, \ldots, J$

\subsection{Internal Pointwise Sensor}

Let us consider the case of pointwise sensor located inside of $\Omega$. The system (46) is augmented with the following output function:

$z(t)=\int_{\Omega} x\left(\xi_{1}, \xi_{2}, t\right) \delta\left(\xi_{1}-b_{1}, \xi_{2}-b_{2}\right) d \xi_{1} d \xi_{2}$

where $b=\left(b_{1}, b_{2}\right)$ is the location of pointwise sensor as defined in (Figure 6).

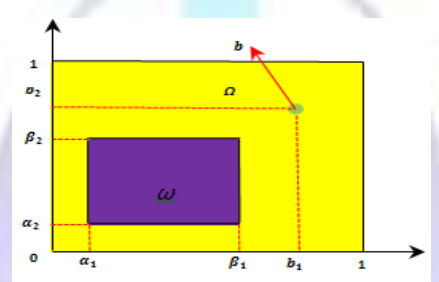

Fig. 6: Rectangular domain $\Omega$, region $\omega$, and location $b$ of internal pointwise sensor.

If $\left(\beta_{1}-\alpha_{1}\right) /\left(\beta_{2}-\alpha_{2}\right) \notin Q$, then $m=1$ and one sensor $\left(b, \delta_{b}\right)$ may be sufficient for $\omega_{E_{G}}$-observability. Then, the dynamical system is given by

$$
\left\{\begin{aligned}
& \frac{\partial y}{\partial t}\left(\xi_{1}, \xi_{2}, t\right)=\Delta y\left(\xi_{1}, \xi_{2}, t\right)+y\left(\xi_{1}, \xi_{2}, t\right) \Theta \\
&+ \mathcal{H}_{\omega}\left(x\left(b_{1}, b_{2}, t\right)-C y(t)\right) \\
& y\left(\xi_{1}, \xi_{2}, 0\right)=y_{0}\left(\xi_{1}, \xi_{2}\right) \Omega \\
& y\left(\eta_{1}, \eta_{2}, t\right)=0
\end{aligned}\right.
$$

Thus, we obtain the following.

Corollary 4.2: The system (46)-(54) is $\omega_{E_{G}}$-observable by the dynamical system (55) if $i\left(b_{1}-\alpha_{1}\right) /\left(\beta_{1}-\alpha_{1}\right)$ and $j\left(b_{2}-\alpha_{2}\right) /\left(\beta_{2}-\alpha_{2}\right) \notin N$, for every $i, 1 \leq i \leq J$.

\subsection{Internal Filament Sensor}

Consider the case where $\Omega=(0,1) \times(0,1)$ and $\omega=\left(\alpha_{1}, \beta_{1}\right) \times\left(\alpha_{2}, \beta_{2}\right) \subset \Omega$. Suppose that the observation on the curve $\sigma=\operatorname{Im}(\gamma)$ with $\gamma \in C^{1}(0,1)$ (Figure 7 ), then we have the following.

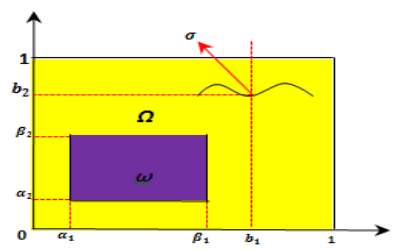

Fig. 7: Rectangular domain $\Omega$, region $\omega$, and location $\sigma$ of internal filament sensors.

Corollary 4.3: If the observation recovered by filament sensor $\left(\sigma, \delta_{\sigma}\right)$ such that it is symmetric with respect to the line $b$, then the system (46)-(54) is $\omega_{E_{G}}$-observable by (55) if $i\left(b_{1}-\alpha_{1}\right) /\left(\beta_{1}-\alpha_{1}\right)$ andj $\left(b_{2}-\alpha_{2}\right) /\left(\beta_{2}-\alpha_{2}\right) \notin N$ for all $i=1, \ldots, J$. 


\subsection{Boundary Zone Sensor}

We consider the system (46) with the Dirichlet boundary conditions and output function (6). We study this case with different geometrical domains.

The domain $(\mathbf{0}, \mathbf{1}) \times(\mathbf{0}, \mathbf{1})$ : now the output function $(2)$ is given by

$z(t)=\int_{\Gamma} \frac{\partial x}{\partial v}\left(\eta_{1}, \eta_{2}, t\right) g\left(\eta_{1}, \eta_{2}\right) d \eta_{1} d \eta_{2}$

Where $\Gamma \subset \partial \Omega$ is the support of the boundary sensor and $g \in L^{2}(\Gamma)$. The sensor $(D, g)$ may be located on the boundary in $\Gamma=\left[\eta_{0_{1}}-I, \eta_{0_{2}}+I\right] \times\{1\},($ Figure 8$)$.

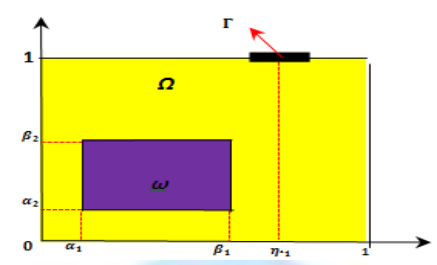

Fig. 8: Rectangular domain and location $\Gamma$ of boundary zone sensor

The dynamical system related to (46)-(56) is given by

$\left\{\begin{array}{r}\frac{\partial y}{\partial t}\left(\xi_{1}, \xi_{2}, t\right)=\Delta y\left(\xi_{1}, \xi_{2}, t\right)+y\left(\xi_{1}, \xi_{2}, t\right) \Theta \\ +\mathcal{H}_{\omega}\left(\left\langle x(., t), g_{i}(.)\right\rangle_{L^{2}\left(\Gamma_{i}\right)}-C y(\xi, t)\right) \\ y\left(\eta_{1}, \eta_{2}, t\right)=0 \\ y\left(\xi_{1}, \xi_{2}, 0\right)=y_{0}\left(\xi_{1}, \xi_{2}\right) \Omega\end{array}\right.$

Which results into:

Corollary 4.4: If the function $g$ is symmetric with respect to $\eta_{1}=\eta_{0_{1}}$. The system (46)-(56) isnot $\omega_{E_{G}}$-observable by the dynamical system (57), if $i\left(\xi_{0_{1}}-\alpha_{1}\right) /\left(\beta_{1}-\alpha_{1}\right) \epsilon N$ for some $i, 1 \leq i \leq J$.

\subsection{Boundary Pointwise Sensor}

Let us consider the system (46) with Dirichlet boundary condition, so, we can study the following. In this case the sensor $\left(b, \delta_{b}\right)$ is located on $\partial \Omega$ (Figure 9$)$. The output function is given by $z(t)=\int_{\partial \Omega} \frac{\partial x}{\partial v}\left(\eta_{1}, \eta_{2}, t\right) \delta\left(\eta_{1}-b_{1}, \eta_{2}-b_{2}\right) d \eta_{1} d \eta_{2}$

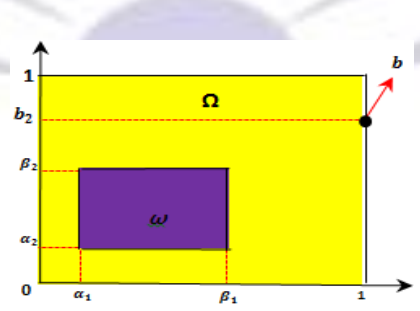

Fig. 9: rectangular domain, and location $b$ of boundary pointwise sensor.

Then we can obtain.

Corollary 5.5: The system (46)-(58) is not $\omega_{E_{G}}$-observable by the system (55) if $i\left(b_{1}-\alpha_{1}\right) /\left(\beta_{1}-\alpha_{1}\right)$ and $j\left(b_{2}-\alpha_{2}\right) /\left(\beta_{2}-\right.$ $\left.\alpha_{2}\right) \in N$, for every $i, i=1, \ldots, J$.

\subsection{Application To One Dimensional Diffusion System}


We can extend previous results to the case of one dimensional system. Consider the following forms

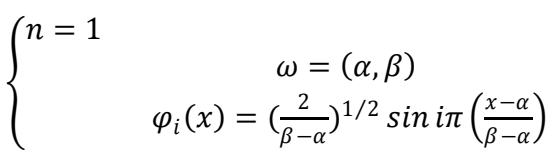

the associated eigenvalues is given by

$$
\left\{\begin{array}{c}
n=1 \\
\omega=(\alpha, \beta) \\
\lambda_{i}=-\frac{i^{2}}{(\beta-\alpha)^{2}} \pi^{2}
\end{array}\right.
$$

In this case system (1) is given by form

$\left\{\begin{array}{l}\frac{\partial x}{\partial t}(\xi, t)=\frac{\partial x}{\partial \xi}(\xi, t)+x(\xi, t) \\ x(\eta, t)=0\end{array}\right.$

Now we discuss the cases in the following below (Figures 10,11, 12):

Corollary 5.1: The system (60)-(2) is $\omega_{E_{G}}$-observable in the following cases:

(1) Case of internal zone sensor must be satisfy: $\frac{x-\alpha}{\beta-\alpha} \notin Q$.

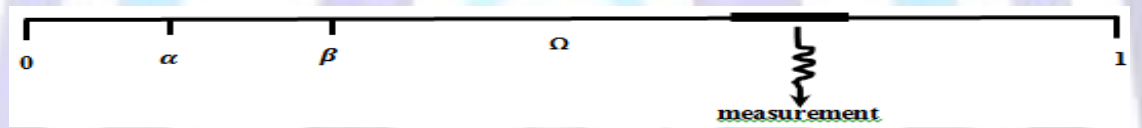

Fig. 10:one-dimensional system with internal zone sensor

(2) Case of internal pointwise sensor must be satisfied: $\frac{b-\alpha}{\beta-\alpha} \notin \mathrm{Q}$.

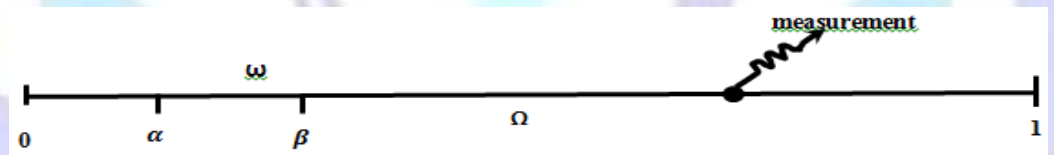

Fig. 11:one-dimensional system with internal pointwise sensor

(3) Case of pointwise boundary sensor must be satisfied: $\frac{b-\alpha}{\beta-\alpha} \notin \mathrm{Q}$.

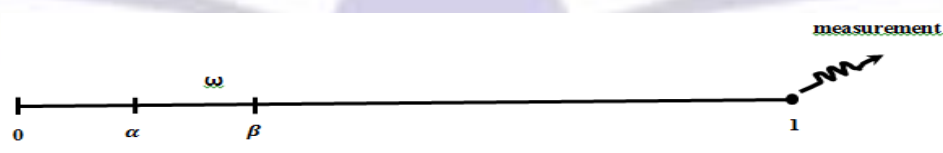

Fig. 12: one-dimensional system with boundary pointwise sensor

Remark 5.2: These results can be extended to the following:

(1) Case of Neumann or mixed boundary conditions [19-20].

(2) Case of disc domain $\Omega=(D, 1)$ and $\omega=\left(0, r_{\omega}\right)$ where $\omega \subset \Omega$ and $0<r_{\omega}<1$ [8].

\section{CONCLUSION}

The original concept developed in this paper is related to sufficient condition of regional exponential general observability in connection with the strategic sensors and locations. Various interesting results concerning the choice of sensor 
structures are given and illustrated in specific situations. Then, we have explained the regional exponential detection problem for a class of distributed parameter systems with regional observability and regional estimator. We have given an extension of exponential regional state reconstruction in the considered sub-region $\omega$ based on the structures of sensors. Thus, we have characterized the existence of such $\omega_{E_{G}}$ - observable (general case). The state to be exponentially estimated on a part of boundary of the domain is under consideration.

\section{ACKNOWLEDGMENTS}

Our thanks in advance to the editors and experts for considering this paper to publish in this estimated journal and for their efforts.

\section{REFERENCES}

[1] Al-Saphory, R. 2001. Asymptotic regional analysis for a class of distributed parameter systems.PhD thesis, University of Perpignan France.

[2] Al-Saphory, R. 2002. Asymptotic regional boundary observer in distributed parameter systems via sensors structures.Sensors, Vol. 2, 137- 152.

[3] Al-Saphory, R. 2011. Strategic sensors and regional exponential observability. ISRN Applied Mathematics. Vol. 2011. Article ID 673052, 1-13.

[4] Al-Saphory, R. Al-Jawari, N.and Al-Janabi A. 2015. Regional gradient strategic sensors characterizations, Journal of Advances in Mathematics. Vol. 10, No. 6, 3604-3618.

[5] Al-Saphory, R. Al-Jawari, N.and Al-Qaisi, A. 2010. Regional gradient detectability for infinite dimensional systems.Tikrit Journal of Pure Science. Vol. 15, No. 2, 1-6.

[6] Al-Saphory, R. Al-Joubory, M. and Jasim, M. 2013. Strategic sensors characterizations. Journal of Mathematical and Computational Science. Vol. 3, No. 2, 401-418.

[7] Al-Saphory, R. Al-Mullah, Sh. 20115.Regional exponential reduced observability in distributed parameter systems.Journal of Advances in Mathematics.Vol. 11, No. 4,5050-5068.

[8] Al-Saphory, R. and El Jai, A. 2001. Sensor structures and regional detectability of parabolic distributes systems. International Journal of Sensors and Actuators. Vol.90,163-171.

[9] Al-Saphory, R. and El Jai, A. 2001. Sensors and regional asymptotic $\omega$-observer for distributed diffusion systems. Sensors, Vol. 1, 161-182.

[10] Al-Saphory, R. and El Jai, A. 2001.Sensors characterizations for regional boundary detectability in distributed parameter systems. Sensors and Actuators A, Vol. 94, No. 1-2, pp. 1-10.

[11] Al-Saphory, R. and EI Jai, A. 2002. Regional asymptotic state reconstruction. International Journal of systems science. Vol. 33, 1025-1037.

[12] Amouroux, M., El Jai, A. and Zerrik, E. 1994. Regional observability of distributed systems. International Journal of Systems Science. Vol. 25, No.2, 301-313.

[13] Borggaard, J. Burns, J. Surana, A. and Zietsman, L. 2009. Control, estimation and optimization of energy efficient buildings, 2009 American Control Conference.Hyatt regency riverfront, St. Louis, MO, USA, MD, USA. June 10-12, 2009.

[14] Curtain, R. F. 1984. Finite-dimensional compensators for parabolic distributed systems with unbounded control and observation. SIAM Journal on Control and Optimization, Vol. 22, No. 2, 255-276.

[15] Curtain, R. F. and Zwart, H. 1995. An introduction to infinite dimensional linear system theory. Springer-Verlag, New York.

[16] El Jai, A. 1991. Distributed systems analysis via sensors and actuators.International Journal of Sensors and Actuators, Vol. 29, No. 1, 1-11.

[17] El Jai, A.and Amouroux, M. 1988. Sensors and observers in distributed parameter systems. International Journal of Control. Vol. 47, No. 1, 333-347.

[18] El Jai, A. and El Yacoubi, S. 1993. On the number of actuators in parabolic system .International Journal of Applied Mathematics and Computer Science. Vol. 103, No. 4, 673-686.

[19] El Jai, A. and Pritchard, A. J. 1987. Sensors and actuators in distributed systems. International Journal of Control, Vol. 46, No. 4, 1139-1153. 
[20] El Jai, A. and Pritchard, A. j. 1988. Sensors and controls in analysis of distributed system. Ellis Harwood series in Mathematics and Applications, Wiley, New York.

[21] El Jai, A.Simon, M.C. and Zerrik, E. 1993.Regional observability and sensor structures.International Journal of Sensor and Actuator. Vol. 39, No. 2, 95-102.

[22] El Jai, A., Simon, M.C. Zerrik, E. and Amouroux, M.1995. Regional observability of a thermal process. IEEE Transaction on Automatic control, Vol. 40, No. 3, 518-521.

[23] El Jai A. Guisset E., Trombe A. and Suleiman A. 2000. Application of boundary observation to thermal systems. In Proceedings of the Fourteenth International Symposium on Mathematical Theory of Networks and Systems, (MTNS 2000) Perpignan France.

[24] Jasim, M. Swady R. and Al-Saphory, R. 2013.Dynamical stability of charged isentropic superdense star model.Journal of Mathematical and Computational Science. Vol. 3, No. 1, 266-277.

[25] Gressang, R. and Lamont, G.1975. Observers for systems characterized by semi-group.IEEE on Automatic and Control.Vol. 20, 523-528.

[26] Luenberger, D. 1966. Observers for multivariable systems. IEEE Transaction on Automatic Control, Vol. 11, $190-197$.

[27] Zerrik, E., Badraoui L. and El Jai, A. 1990. Sensors and regional boundary state reconstruction of parabolic systems. International Journal of Sensors and Actuators, Vol. 75, 102-117.

[28] Zerrik E., Bourray H., and Boutoulout, A. 2002. Regional boundary observability: a numerical approach.internationalJournal of Applied Mathematics and Computer Science, Vol. 12, No. 2, 143-151.

\section{Author's Biographies:}
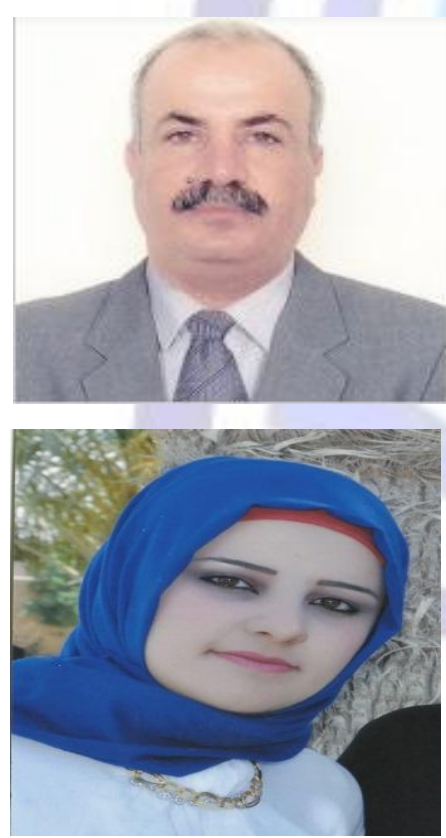

Raheam Al-Saphory is professor associated at the TIKRIT University in IRAQ. He received his Ph.D. degree in Control System and Analysis in (2001) from LTS/ Perpignan University France. He has a post doctorate as a researcher in 2001-2002 at LTS. AlSaphory wrote one book and many papers in the area of systems analysis and control. Also he is a supervisor of many Ph. D. and M. Sc. students and he was Ex-head of Department of Mathematics /College of Education for Pure Science Tikrit University 2010 2011. He visited many centers and Scientific Departments of Bangor University/ Wales/ UK with academic staff of Iraqi Universities in 2013. He is a coordinator of postgraduate studies at Department of Mathematics 2014-present.

Zainab Ali Jaafar is a researcher at the Department of Mathematics/ College of Education for pure Sciences/ TIKRIT University / IRAQ. Here research area focused on Distributed Parameter Systems Analysis and Control. 